Math. Model. Nat. Phenom.

Vol. 8, No. 5, 2013, pp. 31-47

DOI: $10.1051 / \mathrm{mmnp} / 20138503$

\title{
Stability of Traveling Waves in Partly Parabolic Systems
}

\author{
A. Ghazaryan ${ }^{1 *}$, Y. Latushkin ${ }^{2}$, S. Schecter ${ }^{3}$ \\ 1 Department of Mathematics, Miami University, Oxford, OH 45056 USA \\ 2 Department of Mathematics, University of Missouri, Columbia, MO 65211 USA \\ 2 Department of Mathematics, North Carolina State University, Box 8205, Raleigh, NC 27695 USA
}

\begin{abstract}
We review recent results on stability of traveling waves in partly parabolic reactiondiffusion systems with stable or marginally stable equilibria. We explain how attention to what are apparently mathematical technicalities has led to theorems that allow one to convert spectral calculations, which are used in the sciences and engineering to study stability of a wave, into detailed, theoretically-based information about the behavior of perturbations of the wave.
\end{abstract}

Keywords and phrases: traveling wave, spectral stability, linear stability, nonlinear stability, exponential weights

Mathematics Subject Classification: 35K57, 35B35, 47D06

\section{Introduction}

A reaction-diffusion equation in one space-dimension is a partial differential equation of the form

$$
Y_{t}=D Y_{x x}+R(Y)
$$

with $x \in \mathbb{R}, t \geq 0$, and $Y: \mathbb{R} \times \mathbb{R}_{+} \rightarrow \mathbb{R}^{n}$. The function $R: \mathbb{R}^{n} \rightarrow \mathbb{R}^{n}$ is smooth. $D=\operatorname{diag}\left(d_{i}\right)$ is an $n \times n$ constant diagonal matrix with $d_{i}>0$ for $i=1, \ldots, k$, where $k$ a number between 1 and $n$, and $d_{i}=0$ otherwise. The equation is parabolic if $k=n$ and partly parabolic otherwise.

Partly parabolic systems, or, as they are also called in the literature, partly dissipative [56, p.283], partially degenerate [31], or partially parabolic systems, are perhaps less familiar than parabolic systems. They have diffusion in some equations (those for which $d_{i}>0$ ) and no diffusion in others. Examples include equations modeling nerve impulses, such as Hodgkin-Huxley and FitzHugh-Nagumo; combustion and chemical reaction equations in which some reactant is a solid (hence does not diffuse); intracellular calcium dynamics in the presence of immobile buffers [32-34,62,63]; population interaction models in which some populations diffuse and others do not $[11,27,28]$; and models for malignant tumor growth [40].

One direction of study of partly parabolic systems has been existence and properties of attractors of the associated semiflows; see references in [56]. In addition, examples of traveling waves in partly parabolic equations have been studied for a long time. However, interest in traveling waves of partly parabolic

*Corresponding author. E-mail: ghazarar@miamioh.edu

(C) EDP Sciences, 2013 
equations as a class appears to be recent. For commentary on this class of equations, see Tsai [62] and Rottmann-Matthes [48].

Because of Galilean invariance (resulting from no $x$-dependence in the equations), system (1.1) admits solutions of the form $Y_{*}(\xi), \xi=x-c t$, called traveling waves. Traveling waves move with constant velocity while keeping their shape. When $c=0$, they are called standing waves, and are the equilibrium (that is, time-independent) solutions of (1.1). Without loss of generality we will always take $c \geq 0$. In general, traveling waves of (1.1) with velocity $c$ are equilibrium solutions of the PDE

$$
Y_{t}=D Y_{\xi \xi}+c Y_{\xi}+R(Y)
$$

obtained from (1.1) by replacing $x$ by the moving coordinate $\xi=x-c t$. Since a shift $Y_{*}(\cdot+q), q \in \mathbb{R}$, of a traveling wave is also a traveling wave, equilibria of (1.2) come in one-parameter families.

Traveling wave solutions of (1.1) are the simplest solutions other than constants. They arise in applied problems from such fields as optical communication, combustion theory, biomathematics (calcium waves in tissue, nerve conduction, population dynamics), chemistry (autocatalytic reactions), and botany (vegetation patterns). In applications traveling waves are frequently the most important solutions.

Our interest is in traveling waves that asymptotically connect spatially homogeneous states. Hence we suppose that $Y_{*}(\xi)$ is an equilibrium solution of (1.2) with

$$
\lim _{\xi \rightarrow \pm \infty} Y_{*}(\xi)=Y_{ \pm}
$$

$Y_{*}(\xi)$ is called a pulse if $Y_{-}=Y_{+}$and a front if $Y_{-} \neq Y_{+}$. Such traveling waves are found by replacing $Y_{t}$ in (1.2) by 0 , writing the resulting ODE as a first-order system $Z_{\xi}=G(Z, c), Z \in \mathbb{R}^{n+k}$, and looking for solutions that connect equilibria. Equilibria of the ODE represent spatially homogeneous solutions of the PDE.

We shall limit our attention to traveling waves that approach their end states $Y_{ \pm}$exponentially. Such waves are sometimes embedded in a continuum of waves. Some of these waves may not be physical because, for example, they have negative values for physical quantities that must be nonnegative. In other cases some of the waves converge to one or both of their end states at rates slower than exponential. The latter may be stable to exponentially small perturbations of themselves [39], but this means that they are observed only when carefully prepared, nonphysical initial conditions are used. Physically important initial conditions are generally strongly localized and therefore are exponentially small perturbations of traveling waves that converge to their end states exponentially. Hence it is usually sufficient to only consider traveling waves with this property.

In this paper we will review recent, general results that aid in the stability analysis of pulses and fronts in partly parabolic systems.

\section{Linear stability}

\subsection{Definitions}

The definitions in this section do not depend on whether the system is partly or fully parabolic.

Linearizing (1.2) at the traveling wave $Y_{*}$, we obtain the linear PDE

$$
Y_{t}=L Y:=\left(D \partial_{\xi \xi}+c \partial_{\xi}+D R\left(Y_{*}\right)\right) Y .
$$

There are two related constant-coefficient linear PDEs

$$
Y_{t}=L_{ \pm} Y:=\left(D \partial_{\xi \xi}+c \partial_{\xi}+D R\left(Y_{ \pm}\right)\right) Y,
$$

obtained by linearizing (1.2) at $Y_{ \pm}$. The spectrum of the operator associated with $L_{ \pm}$on $L^{2}(\mathbb{R})^{n}$, which we denote $\mathcal{L}_{ \pm}$, can be computed using Fourier transform. It is a collection of curves in the complex plane. 
Definition 2.1. The equilibrium $Y_{-}$is said to be

(i) stable in $L^{2}$ if the spectrum of $\mathcal{L}_{-}$is contained in the half-plane $\{\operatorname{Re} \lambda \leq-\nu\}$ for some $\nu>0$;

(ii) marginally stable in $L^{2}$ if the spectrum of $\mathcal{L}_{-}$is contained in the half plane $\{\operatorname{Re} \lambda \leq 0\}$ and includes at least one point on the imaginary axis;

(iii) unstable in $L^{2}$ if the spectrum of $\mathcal{L}_{-}$contains points with $\operatorname{Re} \lambda>0$; and analogously for $Y_{+}$.

The right-hand boundary of the union of the spectra of $\mathcal{L}_{-}$and $\mathcal{L}_{+}$on $L^{2}$ is also the right-hand boundary of the essential spectrum of the operator associated with $L$ on any of the standard Banach spaces $L^{2}(\mathbb{R})^{n}, L^{1}(\mathbb{R})^{n}, H^{1}(\mathbb{R})^{n}$, or $B U C(\mathbb{R})^{n}$ (bounded uniformly continuous functions); we denote all these operators by $\mathcal{L}$, and we will usually omit the exponent $n$ and the set $\mathbb{R}$ in the notation. The latter two spaces are better suited to the study of nonlinear equations, since they are closed under multiplication.

The discrete spectrum of $\mathcal{L}$ (eigenvalues of finite algebraic multiplicity that are isolated in the spectrum) can be studied using ODE techniques. The approach is based on exponential dichotomies; see [29, Chapter 5], [30], [53]. Briefly, the eigenvalue equation for (2.1), $\lambda U=L U$, can be rewritten as a first-order linear ODE of the form

$$
Z_{\xi}=(B(\xi)+\lambda C) Z, \quad Z: \mathbb{R} \rightarrow \mathbb{R}^{n+k} .
$$

Eigenfunctions $Y(\cdot)$ of $\mathcal{L}$ correspond to solutions $Z(\cdot)$ of $(2.3)$ that lie in the function space under consideration. There is a number $\ell$ such that for $\lambda$ to the right of the essential spectrum of $\mathcal{L}$, the space of solutions of (2.3) that approach 0 exponentially as $\xi=-\infty$ (respectively $\xi=\infty$ ) has dimension $\ell$ (respectively dimension $n+k-\ell$ ). If these spaces have nontrivial intersection, $\lambda$ is in the discrete spectrum of $\mathcal{L}$ on any of the standard function spaces mentioned above; otherwise $\lambda$ is in the resolvent set of $L$. One can construct an analytic function $D(\lambda)$, the Evans function [30,53], defined (at least) to the right of the essential spectrum, whose zeros are the eigenvalues of $\mathcal{L}$; the multiplicity of the zero gives the algebraic multiplicity of the eigenvalue. For the standard function spaces mentioned above, there is always an eigenvalue 0 with eigenfunction $Y_{*}^{\prime}$, the derivative of the traveling wave.

We shall use the following definitions to describe stability properties of the waves:

\section{Definition 2.2.}

(i) A traveling wave $Y_{*}$ is called spectrally stable in a space $\mathcal{E}$ if the spectrum of the linear operator $\mathcal{L}$ on $\mathcal{E}$ is contained in the half-plane $\{\operatorname{Re} \lambda \leq-\nu\}$ for some $\nu>0$, except for a simple eigenvalue at 0 .

(ii) A traveling wave $Y_{*}$ is called spectrally unstable due to essential spectrum in a space $\mathcal{E}$ if the discrete spectrum of the linear operator $\mathcal{L}$ in $\mathcal{E}$ is contained in the half-plane $\{\operatorname{Re} \lambda \leq-\nu\}$ for some $\nu>0$, except possibly for a simple eigenvalue at 0 , but the essential spectrum has nonempty intersection with the imaginary axis.

(iii) Assume that 0 is a simple eigenvalue of $\mathcal{L}$ on a space $\mathcal{E}$, and let $\mathcal{Y}$ denote the null space of the Riesz spectral projection of the operator $\mathcal{L}$ onto the span of $Y_{*}^{\prime}$. In this case, a traveling wave $Y_{*}$ is called linearly stable in $\mathcal{E}$ if $\mathcal{L}$ generates a $C_{0}$ semigroup $e^{t \mathcal{L}}$ that, when restricted to $\mathcal{Y}$, satisfies the estimate $\left\|e^{t \mathcal{L}} \mid \mathcal{Y}\right\| \leq K e^{-\delta t}$ for some $K>0$ and $\delta>0$ and all $t \geq 0$.

(iv) A traveling wave $Y_{*}$ is called nonlinearly stable (or orbitally stable) in $\mathcal{E}$ if a solution of (1.2) that starts near $Y_{*}$ in $Y_{*}+\mathcal{E}$ stays close to the curve of shifts $\left\{Y_{*}(\cdot+q), q \in \mathbb{R}\right\}$ of $Y_{*}$ for $t \geq 0$, in some norm (possibly not the norm of $\mathcal{E}$ ).

(v) A traveling wave $Y_{*}$ is called nonlinearly exponentially (resp. algebraically) stable with asymptotic phase in $\mathcal{E}$ provided (1) it is nonlinearlly stable in $\mathcal{E}$, and (2) a solution of (1.2) that starts near $Y_{*}$ in $Y_{*}+\mathcal{E}$ converges exponentially (resp. algebraically) to a particular shift $Y_{*}\left(\cdot+q_{0}\right)$ of $Y_{*}$ as $t \rightarrow \infty$. The convergence may be in a norm different from the norm on $\mathcal{E}$.

\subsection{Using spectral information to show linear stability}

In the stability theory of traveling waves, one usually attempts to show that spectral stability or linear stability plus some additional conditions imply nonlinear exponential stability with asymptotic phase. Two basic theorems are used: 
1. Henry's Theorem [29, Section 5.1]. If, in the space $\mathcal{E}, R$ defines a $C^{1}$ mapping, the operator $\mathcal{L}$ is sectorial, and the traveling wave $Y_{*}$ is spectrally stable, then $Y_{*}$ is nonlinearly exponentially stable with asymptotic phase.

2. Theorem of Bates and Jones [3, Theorem 1.1 and proof of Theorem 4.3]. If, in the space $\mathcal{E}, R$ defines a $C^{1}$ mapping, and the traveling wave $Y_{*}$ is linearly stable, then $Y_{*}$ is nonlinearly exponentially stable with asymptotic phase.

These theorems are discussed in [53, pp. 1040-1041].

The result of Bates and Jones is proved by showing that in $Y_{*}+\mathcal{E}, Y_{*}$ has a local stable manifold tangent to $\mathcal{Y}$ at $Y_{*}$. Shifting this manifold by $Y(\xi) \rightarrow Y(\xi+q)$ sends it to the stable manifold of the shifted traveling wave $Y_{*}(\xi+q)$. The set of such shifts with $q$ close to 0 foliates a neighborhood of $Y_{*}$, which proves the result.

Henry's result follows from the result of Bates and Jones: the hypotheses of Henry's Theorem imply that $Y_{*}$ is linearly stable, because of a spectral mapping theorem that relates the spectrum of a sectorial operator to the spectrum of the analytic semigroup it generates. However, Henry's proof is different from that of Bates and Jones. First, Henry observes that the hypotheses imply that $Y_{*}$ is linearly stable. Next, Henry decomposes a solution near $Y_{*}$ into $Y(\xi, t)=Y_{*}(\xi+q(t))+\widetilde{Y}(\xi, t)$, with $\widetilde{Y}(\cdot, t) \in \mathcal{Y}$ for each $t$. Using the linear stability of $Y_{*}$, Henry appeals to the variation of constants formula to show that $|q(t)|+\|\widetilde{Y}(t)\|$ stays small, the solution is defined for all time, $\widetilde{Y}(\cdot, t) \rightarrow 0$, and $q(t)$ approaches constant $q_{0}$ as $t \rightarrow \infty$.

In the space $H^{1}$ or $B U C$, Henry's approach applies when the reaction-diffusion equation is parabolic. Bates and Jones, on the other hand, show how to apply their result when the reaction-diffusion equation is partly parabolic, provided the traveling wave is a pulse. The difficulty in using the approach of Bates and Jones is that spectral stability, not linear stability, is the more directly verifiable condition. For operators that generate $C_{0}$ semigroups but are not sectorial, spectral stability does not always imply linear stability. There is no generally applicable spectral mapping theorem; cf. [13] and [16, Section IV.3]. For partly parabolic systems, $\mathcal{L}$ is not sectorial. Indeed, Evans, whose series of papers on stability of pulses in nerve impulse models is now considered fundamental, devoted considerable effort to this issue [17]. His argument was simplified by Bates and Jones. The basic idea is that for a pulse, $\mathcal{L}_{-}=\mathcal{L}_{+}$, so $e^{t \mathcal{L}}$ can be regarded as a compact perturbation of $e^{t \mathcal{L}_{-}}$, a semigroup generated by a constant-coefficient operator.

Henry's result and the result of Bates and Jones are both special cases of general results on existence of locally invariant manifolds and foliations for semilinear equations in Banach spaces. As an example, we mention the following result of Chen, Hale, and Tan [12]. Consider an ordinary differential equation $Y_{t}=A Y+F(Y)$ on a Banach space, with $F$ of class $C^{1}, F(0)=0$ and $D F(0)=0$. Assume (1) $A$ generates a $C_{0}$ semigroup $e^{A t}$ such that the spectrum of $e^{A}$ decomposes into a part on the unit circle and a part bounded away from the unit circle, and (2) $F$ can be restricted to a small neighborhood of 0 and then extended to the whole space in such a way as to have sufficiently small norm. Then there are local center, stable, center-stable, unstable, and center-unstable manifolds of 0 ; there is an invariant foliation of the center-stable manifold such that all solutions that start in one leaf of the foliation converge to the same solution on the center manifold; and there is a similar invariant foliation of the center-unstable manifold. For other results on invariant manifolds see for instance $[4,5,14,37,38,41]$ and the literature cited therein.

A recent result of the authors (see [22]) states that spectral stability implies linear stability of fronts and pulses in partly parabolic systems of the form

$$
\begin{aligned}
\partial_{t} u & =D \partial_{x x} u+\widetilde{A} \partial_{x} u+R_{1}(u, v), \\
\partial_{t} v & =R_{2}(u, v),
\end{aligned}
$$

where $u \in \mathbb{R}^{k}, v \in \mathbb{R}^{n-k}, D$ and $\widetilde{A}$ are constant matrices, $D=\operatorname{diag}\left(d_{1}, \ldots, d_{k}\right)$ with all $d_{i}>0$, and $R_{1}$ and $R_{2}$ continuously differentiable maps. The interesting case is when $1 \leq k<n$, so the equation is partly parabolic. 
The linearization of system (2.4)-(2.5) at a traveling wave with velocity $c$, written in moving coordinates, takes the form

$$
\begin{aligned}
& \partial_{t} U=D \partial_{\xi \xi} U+A \partial_{\xi} U+B_{11}(\xi) U+B_{12}(\xi) V, \\
& \partial_{t} V=c \partial_{\xi} V+B_{21}(\xi) U+B_{22}(\xi) V,
\end{aligned}
$$

where now $A=\tilde{A}+\operatorname{diag}(c, \ldots, c)$. We assume that each $B_{i j}(\xi)$ exponentially approaches a constant matrix $B_{i j}^{ \pm}$as $\xi \rightarrow \pm \infty$, which is the case when the traveling wave approaches its end states exponentially. Consider the linear operator $\mathcal{L}$ associated with the differential expression

$$
L=\left(\begin{array}{cc}
D \partial_{\xi \xi}+A \partial_{\xi}+B_{11} & B_{12} \\
B_{21} & c \partial_{\xi}+B_{22}
\end{array}\right)
$$

on one of the standard Banach spaces $L^{2}(\mathbb{R})^{n}, H^{1}(\mathbb{R})^{n}, L^{1}(\mathbb{R})^{n}$, or $B U C(\mathbb{R})^{n}$.

Theorem 2.3. [22] Suppose the spectrum of $\mathcal{L}$ is contained in $\operatorname{Re} \lambda \leq-\nu, \nu>0$, except for an eigenvalue 0 of finite algebraic multiplicity. Let $\mathcal{Y}$ be the kernel of the Riesz spectral projection onto the generalized eigenspace for the 0 eigenvalue, and let $0<\delta<\nu$. Then there is a number $K>0$ such that $\left\|e^{t \mathcal{L}} \mid \mathcal{Y}\right\| \leq$ $K e^{-\delta t}$.

Theorem 2.3 follows from [22, Theorem 3.1]. It allows one to conclude that if a traveling wave for a partly parabolic problem is spectrally stable, then it is linearly stable. The result of Bates and Jones can then be used to conclude nonlinear exponential stability with asymptotic phase.

The novelty of Theorem 2.3 is twofold. First, it works not only for pulses but also for fronts. As an application, we note that spectral stability for fronts that occur in nerve-impulse equations was proved in $[44,52,69]$. Theorem 2.3 can be used to deduce linear stability from these results; nonlinear exponential stability with asymptotic phase then follows from the result of Bates and Jones. Details are in Section 2.3. Second, Theorem 2.3 is true in $B U C$. A natural space to use in the stability theory of traveling waves would include the waves themselves and patterns that might bifurcate from them. Unlike the other spaces, $B U C$ allows perturbations that are only bounded at infinity; this is sometimes required to capture physically important bifurcating patterns.

We remark that a less traditional class of function spaces that allow bounded perturbations to fronts is the uniformly local spaces introduced in [18] and studied in detail in [42]. Uniformly local spaces have been used to study stability of fronts that undergo a Turing or Hopf bifurcation in the wake of the front in $[8,24]$; they allow one to obtain a priori estimates for the periodic perturbations.

The proof of Theorem 2.3 uses properties of the second-order and first-order operators that are the diagonal elements of the matrix (2.7) (the second-order operator is sectorial, the first-order operator is related to an operator that generates an evolutionary semigroup [13]); triangular factorizations of $\mathcal{L}$; and the Gearhart-Prüss or Greiner Spectral Mapping Theorem [65], for dealing with Hilbert space and Banach space respectively.

In independent work, Jens Rottmann-Matthes, in his Bielefeld thesis [47] under Wolf-Jürgen Beyn, proved a similar spectral stability implies linear stability result. Rottmann-Matthes's approach applies to a more general class of systems, but the analysis is restricted to $H^{1}$, since it uses Laplace transform. His work is based on [35].

Rottmann-Matthes studies the nonlinear system

$$
\begin{aligned}
& \partial_{t} u=D \partial_{x x} u+(g(u, v))_{x}+R_{1}(u, v), \\
& \partial_{t} v=C \partial_{x} v+R_{2}(u, v),
\end{aligned}
$$

where $u \in \mathbb{R}^{k}, v \in \mathbb{R}^{n-k}, D$ and $C$ are constant real matrices, $D+D^{\top}>0, C=\operatorname{diag}\left(c_{1}, \ldots, c_{n-k}\right)$, and $g, R_{1}, R_{2}$ are $C^{3}$; cf. (2.4)-(2.5). He assumes the existence of a steady state solution $Y_{*}$ of (2.8)(2.9), and proves that a Cauchy problem for this system has a unique weak solution that is in fact is 
a strong solution. The solution exists on $0 \leq t<T^{*}$ for some $T^{*} \leq \infty$. For any $T<T^{*},(u, v) \in$ $C\left([0, T], Y_{*}+H^{1}\right) \cap H^{1}\left((0, T), Y_{*}+L^{2}\right)$, and $u$ is also in $L^{2}\left((0, T), Y_{*}+H^{2}\right)$ [47, Theorem 4.7]. The solution is global in the sense that if $T^{*}<\infty$, then the $H^{1}$-norm of $(u, v)$ blows up as $t \rightarrow T^{*}$.

In addition to the nonlinear system (2.8)-(2.9), Rottmann-Matthes considers the linear system

$$
\begin{aligned}
\partial_{t} U & =D \partial_{\xi \xi} U+A_{11}(\xi) \partial_{\xi} U+A_{12}(\xi) \partial_{\xi} V+B_{11}(\xi) U+B_{12}(\xi) V \\
\partial_{t} V & =C \partial_{\xi} V+A_{22}(\xi) \partial_{\xi} V+B_{21}(\xi) U+B_{22}(\xi) V
\end{aligned}
$$

where $D$ and $C$ are as above, $A_{i j}, B_{i j}$ are bounded continuously differentiable matrix valued functions having the limits $A_{i j, \pm}, B_{i j, \pm}$ as $\xi \rightarrow \pm \infty$, and, in addition, $A_{22, \pm}=0$, while the diagonal elements of $B_{22, \pm}$ are strictly negative; see [47, Assumption 4.24]. Compare (2.6). This system is more general than that obtained by linearizing (2.8)-(2.9) about the steady state.

Assuming spectral stability in $H^{1}$ for the linearization of $(2.8)-(2.9)$ at the steady state, RottmannMatthes proves linear stability [47, Theorem 4.34] and nonlinear stability with asymptotic phase [47, Theorem 4.39]. The spectral stability implies linear stability result also holds for the more general linear operator $(2.10)$.

In addition to [47], see [48]-[51].

\subsection{Application of Theorem 2.3 to FitzHugh-Nagumo fronts}

Consider the FitzHugh-Nagumo equation

$$
\begin{aligned}
& u_{t}=u_{x x}+f(u)-v, \\
& v_{t}=\epsilon(u-\gamma v),
\end{aligned}
$$

with $x \in \mathbb{R}, f(u)=u(1-u)(u-a), a \in(0,1)$ fixed. This equation is a simplification of the HodgkinHuxley equation, which models propagation of electrical waves along nerve axons.

The existence of various traveling fronts $\left(u^{*}, v^{*}\right)$, for which $\lim _{\xi \rightarrow \pm \infty}\left(u^{*}(\xi), v^{*}(\xi)\right)$ both exist but are different, has been shown by Yanagida [69] and Deng [15]. The fronts travel at nonzero speeds and approach their limits exponentially as $\xi \rightarrow \pm \infty$.

Let $\left(u^{*}, v^{*}\right)$ be a traveling front solution of (2.11)-(2.12), that is, a stationary solution of the following system obtained from (2.11)-(2.12) by passing to a moving coordinate frame $\xi=x-c t$ :

$$
\begin{aligned}
& u_{t}=u_{\xi \xi}+c u_{\xi}+f(u)-v, \\
& v_{t}=c v_{\xi}+\epsilon(u-\gamma v) .
\end{aligned}
$$

Writing $(u(\xi, t), v(\xi, t))=\left(u^{*}(\xi), v^{*}(\xi)\right)+(U(\xi, t), V(\xi, t))$ and then $W(\xi, t)=(U(\xi, t), V(\xi, t))$, system (2.13)-(2.14) becomes

$$
W_{t}=\mathcal{L} W+\mathcal{N}(W)
$$

with

$$
\begin{gathered}
L W=L(U, V)=\left(\begin{array}{cc}
\partial_{\xi \xi}+c \partial_{\xi}+f^{\prime}\left(u^{*}(\xi)\right) & -1 \\
\epsilon & c \partial_{\xi}-\epsilon \gamma
\end{array}\right)\left(\begin{array}{l}
U \\
V
\end{array}\right) \\
\mathcal{N}(W)=\mathcal{N}(U, V)=\left(\begin{array}{c}
f\left(u^{*}(\xi)+U(\xi)\right)-f\left(u^{*}(\xi)\right)-f^{\prime}\left(u^{*}(\xi)\right) U(\xi) \\
0
\end{array}\right) .
\end{gathered}
$$

One can associate with $L$ a densely defined unbounded linear operator $\mathcal{L}$ on $\mathcal{E}_{0}^{2}, \mathcal{E}_{0}=B U C(\mathbb{R}), L^{2}(\mathbb{R})$, or $H^{1}(\mathbb{R})$. The natural domain is the direct sum of the domains of the operators $\partial_{\xi \xi}$ and $\partial_{\xi}$. The nonlinear operator $\mathcal{N}$ is $C^{1}$ on $\mathcal{E}_{0}^{2}$ for $\mathcal{E}_{0}=B U C(\mathbb{R})$ or $H^{1}(\mathbb{R})$.

For $\mathcal{E}_{0}=B U C(\mathbb{R})$ or $L^{2}(\mathbb{R})$, Yanagida [69], Nii [44], and Sandstede [52] show that $\mathcal{L}$ is spectrally stable. With the aid of [21], it follows that the same is true for $\mathcal{E}_{0}=H^{1}(\mathbb{R})$. Theorem 2.3 and the result of Bates and Jones are used in [22] to obtain the following result. 
Theorem 2.4. ([22]) Let $\mathcal{E}_{0}=B U C(\mathbb{R})$ or $H^{1}(\mathbb{R})$. Then each of the traveling front solutions $\left(u^{*}, v^{*}\right)$ for (2.11)-(2.12) whose existence was shown in Yanagida [69] or Deng [15] is nonlinearly exponentially stable with asymptotic phase in $\mathcal{E}_{0}^{2}$.

\subsection{Marginally unstable spectrum and exponential weight functions}

Returning to the general discussion of (2.1), we note that if the spectrum of $\mathcal{L}_{-}$or $\mathcal{L}_{+}$touches or passes through the imaginary axis then none of the results mentioned in Section 2.2 can be used. However, these spectra sometimes can be shifted to the left of the imaginary axis if a space with a weighted norm is used. For $c>0$, an exponential weight with positive (resp. negative) growth rate shifts the essential spectrum to the left (resp. right).

In the study of traveling waves for viscous conservation laws and related equations, one encounters marginally stable equilibria that cannot be stabilized by any exponential weight. There is now a large body of work by Zumbrun and collaborators on this situation, based on detailed estimates for the semigroup generated by the linearized equations; see, for example, [70].

The idea of using weights was introduced by Sattinger [55]. The weight functions used are of exponential type: for $\alpha=\left(\alpha_{-}, \alpha_{+}\right) \in \mathbb{R}^{2}$, the weight function $\gamma_{\alpha}(\xi)$ is a smoothed version of the function

$$
\gamma_{\alpha}(\xi)= \begin{cases}e^{\alpha_{-} \xi} & \text { if } \xi \leq 0 \\ e^{\alpha_{+} \xi} & \text { if } \xi \geq 0\end{cases}
$$

In this context we denote the original unweighted Banach space $H^{1}$ or $B U C$ by $\mathcal{E}_{0}$, with norm \|\|$_{0}$, and let

$$
\mathcal{E}_{\alpha}=\left\{Y(\xi): \gamma_{\alpha}(\cdot) Y(\cdot) \in \mathcal{E}_{0}\right\},
$$

with norm $\|Y\|_{\alpha}=\left\|\gamma_{\alpha}(\cdot) Y(\cdot)\right\|_{0}$. Associated with $L$ is a linear operator $\mathcal{L}_{\alpha}$ on $\mathcal{E}_{\alpha}$. Via the operator of multiplication by $\gamma_{\alpha}(\cdot)$, the operator $\mathcal{L}_{\alpha}$ is similar to the operator associated with $\gamma_{\alpha} L \gamma_{\alpha}^{-1}$ on $\mathcal{E}_{0}$; spectral information for $\mathcal{L}_{\alpha}$ can be more readily calculated for the latter operator. In particular, $\mathcal{L}_{\alpha-}$ (respectively $\mathcal{L}_{\alpha+}$ ) denotes the operator similar to the constant-coefficient operator $e^{\alpha_{-} \xi} L_{-} e^{-\alpha_{-} \xi}$ (respectively $\left.e^{\alpha_{+} \xi} L_{+} e^{-\alpha_{+} \xi}\right)$, where $L_{ \pm}$are defined by $(2.2)$.

If $\alpha_{-} \leq 0$ and $\alpha_{+} \geq 0$, then $\mathcal{E}_{\alpha}$ is a space of bounded functions (since $H^{1}(\mathbb{R}) \hookrightarrow L^{\infty}(\mathbb{R})$ ), $R$ defines a smooth mapping on $\mathcal{E}_{\alpha}$, and Henry's result or the result of Bates and Jones can be used to prove nonlinear stability. If $\alpha_{-}<0$ (respectively $\alpha_{+}>0$ ), using the space $\mathcal{E}_{\alpha}$ amounts to restricting the allowed perturbations to those that approach $Y_{-}$(respectively $Y_{+}$) like a multiple of $e^{-\alpha_{-} t}$ (respectively $e^{-\alpha_{+} t}$ ) or faster. This may well be a mathematically natural restriction: if one of the end states of the wave is marginally stable or unstable, we may have to restrict our attention to perturbations that approach it at some exponential rate in order to have any chance of a stability result. In some cases, as we shall see, the restriction may also be physically natural.

If $\alpha_{-}>0$ or $\alpha_{+}<0$, then $\mathcal{E}_{\alpha}$ contains unbounded functions, and one cannot use the results of Henry or Bates and Jones to prove stability, since the requirement that $R$ define a $C^{1}$ mapping on $\mathcal{E}_{\alpha}$ is violated. Indeed, such weighted space are not suited to the study of nonlinear problems, since they are not closed under multiplication.

On the other hand, the use of an exponential weight function with $\alpha_{-}>0$ is attractive on physical grounds. With such a weight, a perturbation of a traveling wave that in the sup norm does not decay, or even grows, but lags further and further behind the traveling wave, does not prevent stability. If the state behind the wave is marginally stable, or even unstable, this may well be appropriate.

In physics, a convective instability occurs when perturbations grow in time but are simultaneously transported to the left or right of a traveling wave (but not both) faster than they grow, so that, in a coordinate frame that moves with the wave, they eventually die out at each point in space. By contrast, an absolute instability occurs when perturbations grow at each spatial location in a coordinate frame that moves with the wave. These concepts originated in plasma physics [10]. 
From the mathematical point of view, convective instability is captured by using exponential weights; see $[53,54]$ for references and examples. Indeed, if a perturbation grows while being convected to, for example, $-\infty$, then the perturbation may stay bounded or even decay in an exponentially weighted norm with a weight that decays at $-\infty$.

Such a wave, which is unstable in the space $\mathcal{E}_{0}$ but stable in the space $\mathcal{E}_{\alpha}$, has been called both convectively unstable and convectively stable in the literature. We prefer to call the wave convectively stable, since this terminology stresses the pointwise stability of the wave.

The term "convective" can be informally explained as follows. Suppose that (1.2) has a solution of the form $Y_{*}(\xi)+\tilde{Y}\left(\xi-c_{1} t\right)$ with $c_{1}<0$; thus the traveling wave $Y_{*}(\xi)$ has a perturbation that moves left relative to the wave, and does not decay in an unweighted norm. We ask whether the perturbation decays exponentially in \|\|$_{\alpha}$, where for simplicity $\alpha_{-}=\alpha_{+}>0$. (We need $\alpha_{-}>0$ to get any sort of decay.) We have

$$
\left\|e^{\alpha_{-} \xi} \tilde{Y}\left(\xi-c_{1} t\right)\right\|=\left\|e^{\alpha_{-}\left(\eta+c_{1} t\right)} \tilde{Y}(\eta)\right\|=e^{\alpha_{-} c_{1} t}\left\|e^{\alpha_{-} \eta} \tilde{Y}(\eta)\right\| .
$$

Therefore $\left\|\tilde{Y}\left(\cdot-c_{1} t\right)\right\|_{\alpha}=e^{\alpha_{-} c_{1} t}\|\tilde{Y}\|_{\alpha}$. This is exponential decay because $\alpha_{-} c_{1}<0$.

Definition 2.5. We say that a traveling wave $Y_{*}$ is spectrally convectively stable in the space $\mathcal{E}_{0}$ provided the wave $Y_{*}$ is spectrally unstable in $\mathcal{E}_{0}$ due to the essential spectrum, but there is a pair $\alpha=\left(\alpha_{-}, \alpha_{+}\right) \neq$ $(0,0)$, with $\alpha_{-}, \alpha_{+} \geq 0$, such that the wave $Y_{*}$ is spectrally stable in $\mathcal{E}_{0} \cap \mathcal{E}_{\alpha}$.

Next we propose a corresponding nonlinear definition modeled on Definition 2.2 (v) of nonlinear stability with asymptotic phase.

Definition 2.6. We say that a traveling wave $Y_{*}$ is nonlinearly convectively stable with asymptotic phase in the space $\mathcal{E}_{0}$ provided (1) it is spectrally convectively stable in $\mathcal{E}_{0}$, with $\alpha=\left(\alpha_{-}, \alpha_{+}\right)$; (2) any solution of (1.2) that starts near $Y_{*}$ in $Y_{*}+\left(\mathcal{E}_{0} \cap \mathcal{E}_{\alpha}\right)$ stays near $Y_{*}$ in $Y_{*}+\left(\mathcal{E}_{0} \cap \mathcal{E}_{\alpha}\right)$; and (3) any solution of (1.2) that starts near $Y_{*}$ in $Y_{*}+\left(\mathcal{E}_{0} \cap \mathcal{E}_{\alpha}\right)$ converges in $\mathcal{E}_{\alpha}$ to a particular shift $Y_{*}\left(\cdot+q_{0}\right)$ of $Y_{*}$ as $t \rightarrow \infty$.

Thus Definition 2.6 requires at the linear level instability in $\mathcal{E}_{0}$ due to essential spectrum (Def. 2.2 (ii)) and nonlinear stability with asymptotic phase in $\mathcal{E}_{0} \cap \mathcal{E}_{\alpha}$ in the sense of Definition 2.2 (v). This definition is not standard, but it includes several results in the literature.

Note that the norm of $\mathcal{E}_{0} \cap \mathcal{E}_{\alpha}$ is defined as $\max \left(\|\|_{0},\|\|_{\alpha}\right)$; smallness in $\mathcal{E}_{0} \cap \mathcal{E}_{\alpha}$ is equivalent to smallness in both \|\|$_{0}$ and \|\|$_{\alpha}$. Also, the norm of $\mathcal{E}_{0} \cap \mathcal{E}_{\alpha}$ is equivalent to the norm of $\mathcal{E}_{\beta}$ with $\beta=\left(0, \alpha_{+}\right)$.

The idea of using an exponential weight function with $\alpha_{-}>0$ together with an unweighted norm to prove stability results for traveling waves goes back to a paper of Pego and Weinstein on traveling waves in a dispersive equation [45].

Kunze and Schneider [36] proved a similar type of nonlinear stability of a trivial solution in a model problem with marginally stable equilibria. They assume that perturbations are small in three normsunweighted ( $H^{1}$ or $B U C$ ), weighted, and $L^{1}$ - and show that such perturbations stay small in the unweighted norm and $L^{1}$, and converge - exponentially in the weighted norm and algebraically (like $t^{-\frac{1}{2}}$ ) in the sup norm - to a particular shift of the wave. A part of what Kunze and Schneider show is called diffusive stability with asymptotic phase. A traveling wave is diffusively stable with asymptotic phase if perturbations that are small in both the sup norm and $L^{1}$ stay small in $L^{1}$ and decay like $t^{-\frac{1}{2}}$ in the sup norm to a particular shift of the wave. It is reasonable to expect diffusive stability in parabolic problems with a marginally stable equilibrium. The term "diffusive" is used since the time decay is related to the decay of heat semigroup.

Passing to the exponentially weighted space $\mathcal{E}_{\alpha}$ creates difficulties in applying the results of Henry or of Bates and Jones to derive nonlinear stability from linear stability. Indeed, unless some severe restrictions are imposed, the reaction term $R$ on $\mathcal{E}_{\alpha}$ is no longer $C^{1}$. There is no reason to believe that spectral convective stability implies nonlinear convective stability in general. In all examples that we know of, the passage to nonlinear convective stability requires some additional property of the system. In the work of Pego and Weinstein [45] a Hamiltonian structure is used. In [9,36] a specific reaction term with 
$D R\left(Y_{ \pm}\right)=0$ is considered. In work on Turing and Hopf bifurcation of the left state $[8,24]$ the stability of the bifurcating Turing patterns is used. In the paper [68] on a scalar nonlocal reaction-diffusion equation, properties of the bifurcating periodic pattern are used. In $[19,21,23]$ convective stability is derived using an assumption that the left state has stability for some components of the solution (see Hypotheses 3.1 and 3.2 below).

We shall discuss [23] in more detail, since it applies to a class of partly parabolic systems.

\section{Nonlinear convective stability with asymptotic phase in a class of parabolic and partly parabolic problems}

\subsection{Nonlinear results}

The paper [23] studies a commonly encountered class of problems that includes both parabolic and partly parabolic cases. Somewhat more than nonlinear convective stability with asymptotic phase is proved, as we shall see.

Consider a traveling wave $Y_{*}$ of (1.1), which may be either a front or a pulse. Without loss of generality we take $Y_{-}$to be 0 . Let $\mathcal{E}_{0}=H^{1}(\mathbb{R})$ or $B U C(\mathbb{R})$.

Hypothesis 3.1. Let $\alpha_{-}>0$, and let $\alpha_{+} \geq 0$. We assume that $\alpha_{+}$is small enough so that $Y_{*}^{\prime} \in \mathcal{E}_{\alpha}$, and we assume that $Y_{*}$ is spectrally stable in $\mathcal{E}_{\alpha}$. In appropriate variables we write $Y=(U, V)$, where $U \in \mathbb{R}^{k}, V \in \mathbb{R}^{n-k}$, and we assume that the reaction term $R$ in (1.1) satisfies $R(U, 0)=0$.

Many combustion problems satisfy this hypothesis. For example, in a combustion problem with $n-1$ independent reactants, let $y_{1}$ denote temperature and let $\left(y_{2}, \ldots, y_{n}\right)$ denote reactant concentrations. Suppose the left state of a combustion front with positive velocity has temperature $y_{1}=y_{1-}>0$ and reactant concentrations $\left(y_{2}, \ldots, y_{n}\right)=(0, \ldots, 0)$ (i.e., behind the front temperature is high and the reactants are all burned). Let $U=u$ and $V=\left(v_{1}, \ldots, v_{n-1}\right)$ with $u=y_{1}-y_{1-}$ and $\left(v_{1}, \ldots, v_{n-1}\right)=$ $\left(y_{2}, \ldots, y_{n}\right)$. Then $Y_{-}=0$. Since the reaction rate is 0 when the reactant concentrations are all 0 , the reaction term will have the appropriate form $R(U, 0)=0$ mentioned in Hypothesis 3.1.

Referring to the general equation (1.1), for $Y=(U, V) \in \mathbb{R}^{n}$, where $U \in \mathbb{R}^{k}, V \in \mathbb{R}^{n-k}$, and the splitting is such that $R(U, 0)=0$, equation (1.1) takes the form

$$
\begin{aligned}
& U_{t}=D_{1} U_{x x}+R_{1}(U, V), \\
& V_{t}=D_{2} V_{x x}+R_{2}(U, V),
\end{aligned}
$$

with $D_{1}$ and $D_{2}$ nonnegative diagonal matrices, and $R_{1}(U, 0)=R_{2}(U, 0)=0$. Linearizing $(1.2)$ at $Y_{-}=(0,0)$, we obtain

$$
\begin{aligned}
U_{t} & =L^{(1)} U+D_{2} R_{1}(0,0) V:=D_{1} U_{\xi \xi}+c U_{\xi}+D_{2} R_{1}(0,0) V, \\
V_{t} & =L^{(2)} V:=D_{2} V_{\xi \xi}+c V_{\xi}+D_{2} R_{2}(0,0) V .
\end{aligned}
$$

Note the triangular structure of the linearization at $Y_{-}$.

Hypothesis 3.2. We assume that the operator associated with $L^{(2)}$ on $\mathcal{E}_{0}$ has its spectrum in the halfplane $\operatorname{Re} \lambda \leq-\rho$ for some $\rho>0$.

On the other hand, the spectrum of $L^{(1)}$ on $\mathcal{E}_{0}$ is the set of curves $\lambda=d_{j} \nu^{2}+c i \nu, \nu \in \mathbb{R}, j=1, \ldots$, $k$. Each curve touches (if $d_{j}>0$ ) or equals (if $d_{j}=0$ ) the imaginary axis, so the equilibrium $Y_{-}$is marginally stable. Note, however, that in $\mathcal{E}_{0}$ the operator associated with $L^{(1)}$ generates a semigroup that is uniformly bounded for $t \geq 0$. (For $d_{j}>0$ this can be seen by explicitly writing the semigroup using the heat kernel; for $d_{j}=0$ the semigroup preserves the norm.) 
Theorem 3.3. Assume Hypotheses 3.1 and 3.2. Then the wave $Y_{*}$ is nonlinearly convectively stable in the sense of Def. 2.6, with a given by Hypothesis 3.1. Thus if the perturbation $(\widetilde{U}(\cdot, 0), \widetilde{V}(\cdot, 0))$ of the traveling wave is initially small in $\mathcal{E}_{0} \cap \mathcal{E}_{\alpha}$, then the corresponding solution of (1.2) decays exponentially in $\mathcal{E}_{\alpha}$ as $t \rightarrow \infty$ to a particular shift of the wave. The solution can be written as

$$
(U, V)(\xi, t)=\left(U_{*}(\xi+q(t))+\widetilde{U}(\xi, t), V_{*}(\xi+q(t))+\widetilde{V}(\xi, t)\right)
$$

where, for each $t$, the function $(\widetilde{U}(\cdot, t), \widetilde{V}(\cdot, t))$ belongs to a fixed subspace of $\mathcal{E}_{0} \cap \mathcal{E}_{\alpha}$ complementary to the the span of $Y_{*}^{\prime}(\cdot)$. $\widetilde{U}(\cdot, t)$ stays small in $\mathcal{E}_{0}$, while $\widetilde{V}(\cdot, t)$ decays exponentially in $\mathcal{E}_{0}$ as $t \rightarrow \infty$.

Thus in the unweighted norm the $U$-component of a perturbation stays small and the $V$-component decays. More can be said about the $U$-component if the diagonal entries of $D_{1}$ are all positive.

Theorem 3.4. In addition to the assumptions in Theorem 3.3, let us suppose that the linear equation $U_{t}=L^{(1)} U$ is parabolic, i.e., the diagonal entries of $D_{1}$ are all positive. If the initial perturbation of the traveling wave is also small in $L^{1}$, then $\widetilde{U}(\cdot, t)$ stays small in the $L^{1}$-norm and decays like $t^{-\frac{1}{2}}$ in the $L^{\infty}$-norm as $t \rightarrow \infty$.

This sort of decay (diffusive stability) is typical for the heat equation.

These theorems are useful for both parabolic and partly parabolic systems. We will discuss applications to partly parabolic systems only.

The proofs of Theorems 3.3 and 3.4 use Theorem 2.3 in order to include the possibility that $D_{1}$ and $D_{2}$ may have some diagonal entries equal to 0 .

\subsection{Application to gasless combustion [21].}

Theorems 2.3, 3.3, and 3.4 are applicable to the following simple combustion model in one space dimension, which originally motivated our work:

$$
\begin{aligned}
& \partial_{t} y_{1}=\partial_{x x} y_{1}+y_{2} \rho\left(y_{1}\right), \\
& \partial_{t} y_{2}=-\beta y_{2} \rho\left(y_{1}\right),
\end{aligned}
$$

where

$$
\rho\left(y_{1}\right)= \begin{cases}e^{-\frac{1}{y_{1}}} & \text { if } y_{1}>0 \\ 0 & \text { if } y_{1} \leq 0\end{cases}
$$

In this system, $y_{1}$ is temperature, $y_{2}$ is concentration of unburned fuel, $\rho$ is unit reaction rate, and $\epsilon$ and $\beta>0$ are constant parameters. Our motivation for looking at this well-studied problem, in which the reactant does not diffuse, was heat-enhanced methods of oil recovery in which the reactant is coke contained in the rock formation [1].

The value $y_{1}=0$ represents ignition temperature and is also taken to be the background temperature, at which the reaction does not take place. If one looks for traveling waves $\left(y_{1 *}, y_{2 *}\right)$ such that $\left(y_{1 *}(-\infty), y_{2 *}(-\infty)\right)=\left(y_{1-}, 0\right)$ with $y_{1-}>0,\left(y_{1 *}(\infty), y_{2 *}(\infty)\right)=(0,1)$, and $\left(y_{1 *}(\xi), y_{2 *}(\xi)\right)$ approaches its ends states exponentially as $\xi \rightarrow \pm \infty$, then one finds that for each $\beta$ there is a unique $c>0$ for which such a wave exists. This wave represents a combustion front that leaves behind it high temperature $y_{-}=1 / \beta$ and no fuel, while in front of it temperature is 0 and there is fuel, with concentration normalized to 1 .

The lack of diffusion in the second equation inspired the linear Theorem 2.3, and the form of the nonlinear term in this and related problems inspired Theorems 3.3 and 3.4.

Both equilibria $(1 / \beta, 0)$ and $(1,0)$ are marginally stable, but the left equilibrium has stability of one component, as Theorem 3.3 requires. More precisely, the linearization about $(1 / \beta, 0)$, in the frame $\xi=x-c t$ that moves with the front, is

$$
\begin{aligned}
& \partial_{t} y_{1}=\partial_{\xi \xi} y_{1}+c \partial_{\xi} y_{1}+\rho(1 / \beta) y_{2}, \\
& \partial_{t} y_{2}=c \partial_{\xi} y_{2}-\beta \rho(1 / \beta) y_{2} .
\end{aligned}
$$


Note that the second equation is decoupled from the first, and the differential operator defined by the second equation on an unweighted space has its spectrum strictly to the left of the imaginary axis.

For the full system in the moving frame, the spectrum of the linearization on an unweighted space at $\left(y_{-}, 0\right)$ is a parabola in the left-half plane that touches the origin (caused by the first equation), together with a vertical line to the left of the imaginary axis (caused by the second equation). At $(0,1)$ the spectrum is the same parabola and the imaginary axis.

To move the spectrum of the linearization at these points to the left, one needs a weight function $\gamma_{\alpha}(\xi)$ as in (2.16) with $\alpha_{-}$and $\alpha_{+}$both positive. We simply use the function $\gamma_{\alpha}(\xi)=e^{\alpha \xi}$ with $\alpha$ positive but not too big.

An a priori upper bound for the modulus of isolated eigenvalues was obtained in [67]. With the aid of this bound, a numerical Evans function calculation in [2] shows that for small $\beta$ there are no eigenvalues in the right half-plane other than 0 , which was shown numerically to be simple. For larger $\beta$ the traveling wave apparently loses stability when a pair of complex eigenvalues crosses the imaginary axis (see numerical evidence in [6]). In [21] we verified analytically that the 0 eigenvalue is simple for all $\beta$.

After the change of variables $u=y_{1}-y_{1-}, v=y_{2}$, one can apply the nonlinear theorems formulated above. The conclusion is that if one makes a perturbation of the wave that is small in both the unweighted norm $\left(H^{1}\right.$ or $\left.B U C\right)$ and the corresponding weighted norm with weight function $e^{\alpha \xi}$, then the perturbed initial condition will decay in the weighted norm to a shift of the wave, while in the unweighted norm the $u$-component of the perturbation will remain small, and the $v$-component of the perturbation will decay exponentially. If the $u$-component of the perturbation is in addition small in $L^{1}$, it will decay in the sup norm like $t^{-\frac{1}{2}}$ as $t \rightarrow \infty$.

All these conclusions have natural physical interpretations, which we will now briefly discuss.

(1) Since $\alpha>0$, we restrict ourself only to perturbations that decay in space exponentially ahead of the front. In $B U C$, some restriction of this sort is clearly necessary, since (3.3)-(3.4) admits fronts that decay in space very slowly to the right state; they cannot be allowed as perturbations. Nor can perturbations be allowed that approach a right state $\left(0, y_{2+}\right)$ with $y_{2+} \neq 1$.

(2) Physically natural initial conditions have $\left(y_{1}, y_{2}\right)=(0,1)$ on an interval $x_{0} \leq x<\infty$. On this interval they constitute an exponentially small perturbation of the front. Hence we can expect that physically natural initial conditions will produce the front that converges to its rest states exponentially, not one of the fronts that converge more slowly. This is in fact what is seen in simulations.

(3) Since $\alpha>0$, perturbations can be left behind the traveling wave without decay in the unweighted norm; in the moving variable $\xi$, in the weighted norm, they are gradually killed by multiplication by $e^{\alpha \xi}$. For example, imagine initial conditions in which the fuel $y_{2}$ is 0 for $x<-\epsilon$ and 1 for $x>\epsilon$, and there is some heat just near $x=0$. If there is enough heat, combustion will start near $x=0$ and a combustion wave will propagate to the right, where the fuel is. The heat produced will gradually diffuse. Such a solution will eventually look like our traveling wave at the right but not far to the left (where the $y_{1}$-component of the traveling wave approaches $\frac{1}{\beta}$ but the $y_{1}$-component of the solution approaches 0 ). This is convergence in the weighted norm. The initial heat perturbation of the traveling wave was not in $L^{1}$, so Theorem 3.4 does not apply.

(4) If a bump of heat is added behind the traveling wave, where there is no fuel to burn, it will diffuse according to the heat equation. This is consistent with Theorem 3.4.

(5) On the other hand, if a bump of fuel is added behind the traveling wave, where the temperature is high, it will immediately burn. This is consistent with the exponential decay of $v$ predicted by Theorem 3.3 .

Thus Theorems 3.3 and 3.4, applied to the combustion model (3.3)-(3.4), do not just "make rigorous" the formal argument for stability of the traveling wave based on the spectrum of the linearization. In addition, they use the spectral calculations commonly done in science and engineering to obtain detailed, physically natural information about the stability of the wave. 
The results can be viewed as an example of "front propagation into unstable states" [66]. In a wave traveling to the right, the left state gradually takes over from the right state. It is natural to expect that for stability, the left state must be stable, but the right state need not be. In the combustion example, the left state has a sort of stability (its spectrum touches the imaginary axis), and the right state has less (its spectrum includes the imaginary axis). The lack of stability of the right state is taken into account by only allowing certain perturbations at the right, which nevertheless constitute a physically important class of perturbations. The marginal stability of the left state translates into the actual behavior of the allowed perturbations.

\subsection{Application to exothermic-endothermic chemical reactions [23]}

Theorem 3.3 is also applicable to a model in which two chemical reactions occur at rates determined by temperature [58-60]. One reaction is exothermic (produces heat), the other is endothermic (absorbs heat). In the original model, both reactants and heat can diffuse.

The system considered in [58-60] reads

$$
\begin{aligned}
& \partial_{t} z_{1}=\partial_{x x} z_{1}+z_{2} f_{2}\left(z_{1}\right)-\sigma z_{3} f_{3}\left(z_{1}\right) \\
& \partial_{t} z_{2}=d_{2} \partial_{x x} z_{2}-z_{2} f_{2}\left(z_{1}\right) \\
& \partial_{t} z_{3}=d_{3} \partial_{x x} z_{3}-\tau z_{3} f_{3}\left(z_{1}\right) .
\end{aligned}
$$

Here $z_{1}$ is temperature, $z_{2}$ is quantity of an exothermic reactant, and $z_{3}$ is quantity of an endothermic reactant. The parameters $\sigma$, and $\tau$ are positive, and there are positive constants $a_{i}$ and $b_{i}$ such that

$$
f_{i}(u)= \begin{cases}a_{i} e^{-\frac{b_{i}}{u}} & \text { for } u>0 \\ 0 & \text { for } u \leq 0\end{cases}
$$

The parameters $d_{2}, d_{3}$ in [58-60] are assumed to be positive, but the nonlinear stability results formulated below hold if either or both of $d_{2}, d_{3}$ are 0 .

Let $Z_{*}$ be a traveling wave solution of (3.5)-(3.7) with speed $c>0, Z_{-}=\left(1-\frac{\sigma}{\tau}, 0,0\right)$, and $Z_{+}=$ $(0,1,1)$. In [58], Simon, et al., show numerically that in certain parameter regimes, such traveling waves exist for which both end states are approached at an exponential rate.

Making the change of variables $y_{1}=z_{1}-\left(1-\frac{\sigma}{\tau}\right), y_{2}=z_{2}, y_{3}=z_{3}$, and passing to the moving coordinate frame $\xi=x-c t$, converts $(3.5)-(3.7)$ to the system

$$
\begin{aligned}
\partial_{t} y_{1} & =\partial_{\xi \xi} y_{1}+c \partial_{\xi} y_{1}+y_{2} f_{2}\left(z+y_{1}\right)-\sigma y_{3} f_{3}\left(z+y_{1}\right), \\
\partial_{t} y_{2} & =d_{2} \partial_{\xi \xi} y_{2}+c \partial_{\xi} y_{2}-y_{2} f_{2}\left(z+y_{1}\right) \\
\partial_{t} y_{3} & =d_{3} \partial_{\xi \xi} y_{3}+c \partial_{\xi} y_{3}-\tau y_{3} f_{3}\left(z+y_{1}\right) .
\end{aligned}
$$

Applying Theorem 3.3 to this system yields the following detailed stability result. Suppose the constants $d_{2}, d_{3}, \sigma, \tau, a_{i}, b_{i}$, and $c>0$ are chosen so that there is a traveling wave $Y_{*}$ that approaches 0 exponentially as $\xi \rightarrow-\infty$ and approaches $Y_{+}=(-z, 1,1), z=1-\frac{\sigma}{\tau}>0$, exponentially as $\xi \rightarrow \infty$. Assume also that this wave has no unstable isolated eigenvalues. Let $\alpha=\left(\alpha_{-}, \alpha_{+}\right), \alpha_{-}>0$ and $\alpha_{+}>0 ; \beta=\left(0, \alpha_{+}\right)$, and $\mathcal{E}_{0}=H^{1}(\mathbb{R})$ or $B U C(\mathbb{R})$. Let $Y^{0} \in Y_{*}+\mathcal{E}_{\beta}^{3}$ with $\left\|Y^{0}-Y_{*}\right\|_{\beta}$ small, and let $Y(t)$ be the solution of the system (3.8)-(3.10) in $Y_{*}+\mathcal{E}_{\beta}^{3}$ with $Y(0)=Y^{0}$. Then:

1. $Y(t)$ is defined for all $t \geq 0$.

2. $Y(t)=\tilde{Y}(t)+Y_{*}(\xi-q(t))$ with $\tilde{Y}(t)$ in a fixed subspace of $\mathcal{E}_{\beta}^{3}$ complementary to the span of $Y_{*}^{\prime}$.

3. $\|\tilde{Y}(t)\|_{\beta}+|q(t)|$ is small for all $t \geq 0$.

4. $\|\widetilde{Y}(t)\|_{\alpha}$ decays exponentially as $t \rightarrow \infty$.

5. There exists $q^{*}$ such that $\left|q(t)-q^{*}\right|$ decays exponentially as $t \rightarrow \infty$.

6. There is a constant $C$ independent of $Y^{0}$ such that $\left\|\tilde{y}_{1}(t)\right\|_{0} \leq C\left\|\widetilde{Y}^{0}\right\|_{\beta}$ for all $t \geq 0$. 
7. $\left\|\left(\tilde{y}_{2}, \tilde{y}_{3}\right)(t)\right\|_{0}$ decays exponentially as $t \rightarrow \infty$.

The conclusions of this theorem can be interpreted physically as in Section 3.2. This is another example in which a numerical study of the Evans function can be coupled with a rather routine check of the hypotheses of Theorem 3.3 to produce quite detailed information on the kind of stability that the traveling wave enjoys.

\subsection{Application to gasless combustion with heat loss [57]}

The following model is an extension of (3.3)-(3.4):

$$
\begin{aligned}
\partial_{t} y_{1} & =\partial_{x x} y_{1}+y_{2} \rho\left(y_{1}-\bar{y}_{1}\right)-\gamma y_{1}, \\
\partial_{t} y_{2} & =\kappa \partial_{x x} y_{2}-\beta y_{2} \rho\left(y_{1}-\bar{y}_{1}\right),
\end{aligned}
$$

with $\beta$ and $\rho$ as before. Here $\kappa \geq 0$ is the diffusion constant of the reactant, $\bar{y}_{1} \geq 0$ is ignition temperature, $y_{1}=0$ is background temperature, and $-\gamma y_{1}, \gamma \geq 0$, represents heat loss to the environment due to Newton's law of cooling.

There is an extensive literature on this system in the case $\kappa>0$; see [61] for a review. Equilibria must have $y_{1}=0$. If $y_{2}$ is normalized to be 1 ahead of a possible combustion front, so the right state of the front is $(0,1)$, then it turns out that the left state of the front is $\left(0, y_{1}^{*}\right)$, where $y_{1}^{*}>0$ represents the unknown concentration of reactant left unburned. For fixed $(\kappa, \beta)$ and small $\gamma>0$, there are two combustion fronts, a somewhat mysterious one with speed near 0 (mysterious in the sense that its speed goes to 0 as $\gamma \rightarrow 0$, but the bifurcation at $\gamma=0$ has not been successfully analyzed), and one that is related to the known combustion front for $\gamma=0$. Existence of the two waves has been proved for some values of $(\kappa, \beta)$ by Leray-Schauder degree, and is known numerically [7,46]. In addition, "high activation-energy asymptotics" has been used to study the limit $\beta \rightarrow \infty$, i.e., the limit in which the reaction produces less and less heat. As $\gamma$ increases, the two waves appear to join and disappear in a saddle-node bifurcation [57]. Spectral stability of the waves has been studied for $\kappa>0$ [57] and $\kappa=0$ [25] by identifying the essential spectrum and, via numerical Evans function calculations, the eigenvalues.

In [25], geometric singular perturbation theory (instead of Leray-Schauder degree or asymptotics) is used to show existence of traveling waves with speeds near the speeds of the waves that exist for $\gamma=0$. In some parameter regimes numerical Evans function calculation shows that the waves are spectrally unstable due to essential spectrum only.

In this case linear and nonlinear results from [22] and [23] can be applied to prove stability for both $\kappa=0$ and $\kappa>0$.

One interest of this work is that, when applying Theorems 3.3 and 3.4, the variables that play the roles of $u$ and $v$ are reversed in the case $\gamma>0$ from their previous roles in the case $\gamma=0$. Hence these results say that if a little heat is added behind the wave, it will quickly vanish, but if a little reactant is added behind the wave, it may not. The reason is that in the case of heat loss to the environment, the temperature far back in the wave is near 0; added heat is lost to the environment, and added reactant does not burn.

\section{Some open problems in the stability analysis of waves in partly parabolic systems}

\subsection{Porous medium combustion}

The following well-known, simplified model of combustion in a porous medium is due to Sivashinsky; see the review paper [26] for references. 


$$
\begin{aligned}
T_{t}-\left(1-\gamma^{-1}\right) P_{t} & =\epsilon T_{x x}+Y G(T), \\
P_{t}-T_{t} & =P_{x x}, \\
Y_{t} & =\epsilon L e^{-1} Y_{x x}-\gamma Y G(T) .
\end{aligned}
$$

Here $P, T$ and $Y$ are the appropriately scaled pressure, temperature, and concentration of the deficient reactant, $\gamma>1$ is the specific heat ratio, $\epsilon$ is the ration of thermal to molecular diffusivities, and $G(T)$ is a function of combustion type with ignition temperature. More precisely, $G(T)=0$ for $0 \leq T<T_{\text {ign }}$ and is an increasing Lipschitz continuous function for $T \geq T_{i g n}$, except for a possible discontinuity at the ignition temperature $T=T_{i g n}$. For $\epsilon=0$, it is known that there is a unique combustion front that connects the completely burned state to the state in which all of the fuel is present. It is also known that for small $\epsilon>0$, this front perturbs to a unique front with nearby speed. In both cases the stability properties of the front are not known.

For $\epsilon>0$, the system can be put into a more standard form by means of the linear transformation

$$
\begin{aligned}
T & =\frac{-\gamma+\gamma \epsilon+\mu(\gamma, \epsilon)}{2 \gamma \epsilon} \tilde{T}+\frac{-\gamma+\gamma \epsilon-\mu(\gamma, \epsilon)}{2 \gamma} \tilde{P}, \quad \mu(\gamma, \epsilon)=\sqrt{\gamma^{2}+2 \gamma^{2} \epsilon+\gamma^{2} \epsilon^{2}-4 \gamma \epsilon}, \\
P & =\tilde{T}+\epsilon \tilde{P} \\
Y & =\tilde{Y} .
\end{aligned}
$$

The resulting system is

$$
\begin{aligned}
\tilde{T}_{t} & =\frac{(\gamma+\gamma \epsilon+\mu(\gamma, \epsilon))}{2} \tilde{T}_{x x}+\frac{\gamma(\gamma+\gamma \epsilon+\mu(\gamma, \epsilon))}{2 \mu(\gamma, \epsilon)} \mathcal{G}(\tilde{T}, \tilde{P}, \tilde{Y}), \\
\tilde{P}_{t} & =\frac{(\gamma+\gamma \epsilon-\mu(\gamma, \epsilon))}{2} \tilde{P}_{x x}-\frac{\gamma(\gamma+\gamma \epsilon-\mu(\gamma, \epsilon))}{2 \epsilon \mu(\gamma, \epsilon)} \mathcal{G}(\tilde{T}, \tilde{P}, \tilde{Y}), \\
\tilde{Y}_{t} & =\frac{\epsilon}{L e} \tilde{Y}_{x x}-\gamma \mathcal{G}(\tilde{T}, \tilde{P}, \tilde{Y}),
\end{aligned}
$$

where

$$
\mathcal{G}(\tilde{T}, \tilde{P}, \tilde{Y})=\tilde{Y} G\left(\frac{-\gamma+\gamma \epsilon+\mu(\gamma, \epsilon)}{2 \gamma \epsilon} \tilde{T}+\frac{-\gamma+\gamma \epsilon-\mu(\gamma, \epsilon)}{2 \gamma} \tilde{P}\right) .
$$

For $\epsilon>0$ fixed and $L e \gg 1$, the diffusion coefficient in (4.6) is small. In the limit $L e=\infty,(4.4)-(4.6)$ is partly parabolic.

On the other hand, (4.1)-(4.3) is often simplified by taking $\epsilon=0$. In this case, introducing a new variable $R=T-\left(1-\gamma^{-1}\right) P$ leads to the equivalent, partly parabolic system,

$$
\begin{aligned}
R_{t} & =Y G\left(R+\left(1-\gamma^{-1}\right) P\right), \\
P_{t} & =\gamma P_{x x}+\gamma Y G\left(R+\left(1-\gamma^{-1}\right) P\right), \\
Y_{t} & =-\gamma Y G\left(R+\left(1-\gamma^{-1}\right) P\right),
\end{aligned}
$$

where the first two equations are decoupled from the third.

When $G(T)$ is a smooth function that equals 0 for $0 \leq T \leq T_{i g n}$ and is strictly increasing for $T \geq T_{i g n}$, both systems (4.4)-(4.6) and (4.7)-(4.9) satisfy the assumption on the reaction term in Hypothesis 3.1, provided $U$ is the first two variables and $V$ is the third. With this splitting, Hypothesis 3.2 is also satisfied. However, it is not known whether there is a region in parameter space where the spectral stability condition of Hypothesis 3.1 holds. The essential spectrum is not a problem, but it is not known whether discrete eigenvalues always prevent stability. 


\subsection{Simplified Model of Calcium Dynamics}

In [64] a simplified model of intracellular calcium dynamics is proposed:

$$
\begin{aligned}
\partial_{t} u & =D \partial_{x x} u+F(u, w)+\epsilon\left(J_{i n}-k_{m} u\right), \\
\partial_{t} w & =-\gamma F(u, w),
\end{aligned}
$$

with

$$
\begin{aligned}
F(u, w) & =f(u)(w-u)-k_{s} u:=f(u) w-g(u), \\
f(u) & =\alpha+k_{f} \frac{u^{2} \phi_{2}}{\left(u^{2}+\phi_{1}^{2}\right)\left(u+\phi_{2}\right)}, \\
g(u) & =f(u) u+k_{s} u,
\end{aligned}
$$

where $J_{i n}$ is a bifurcation parameter, and $D, \epsilon, k_{m}, k_{f}, k_{s}, \phi_{1}, \phi_{2}$ and $\gamma$ are positive constants with appropriate physical meanings. A variety of traveling waves has been found, and their spectral stability has been studied in [64] using the Evans function. There are parameter regimes where the wave is spectrally unstable due to the essential spectrum. It is not known what kind of instability is created. As written, the system does not satisfy the assumption on the reaction term in Hypothesis 3.1.

Acknowledgements. While working on this paper, Schecter was supported by the National Science Foundation under grant DMS-1211707, Latushkin was supported by the NSF grant DMS-1067929 and by the Research Board and Research Council of the University of Missouri, and Ghazaryan was supported by Simons Foundation's Collaboration Grant for Mathematicians. Ghazaryan also want to express her sincere thanks to the IMA for the hospitality during her stay in Fall 2012 on occasion of the Annual Thematic Program on Infinite Dimensional and Stochastic Dynamical Systems and Their Applications.

\section{References}

[1] I. Y. Akkutlu, Y. C. Yortsos. The dynamics of in-situ combustion fronts in porous media. Combustion and Flame, 134 (2003), 229-247.

[2] S. Balasuriya, G. Gottwald, J. Hornibrook, S. Lafortune. High Lewis number combustion wavefronts: a perturbative Melnikov analysis. SIAM J. Appl. Math., 67 (2007), 464-486.

[3] P. W. Bates, C. K. R. T. Jones. Invariant manifolds for semilinear partial differential equations. Dynamics Reported 2, 1-38, Dynam. Report. Ser. Dynam. Systems Appl. vol. 2. Wiley, Chichester, 1989.

[4] P. Bates, K. Lu, C. Zeng. Invariant foliations near normally hyperbolic invariant manifolds for semiflows. Trans. Amer. Math. Soc., 352 (2000), 4641-4676.

[5] P. Bates, K. Lu, C. Zeng. Existence and persistence of invariant manifolds for semiflows in Banach space. Mem. Amer. Math. Soc., 135 (1998), no. 645.

[6] A. Bayliss, B. Matkowsky. Two routes to chaos in condensed phase combustion. SIAM J. Appl. Math., 50 (1990), 437-459.

[7] V. Giovangigli. Nonadiabatic plane laminar flames and their singular limits. SIAM J. Math. Anal., 21 (1990), 13051325.

[8] M. Beck, A. Ghazaryan, B. Sandstede. Nonlinear convective stability of travelling fronts near Turing and Hopf instabilities. J. Differential Equations, 246 (2009), 4371-4390.

[9] T. Brand, M. Kunze, G. Schneider, T. Seelbach. Hopf bifurcation and exchange of stability in diffusive media. Arch. Ration. Mech. Anal., 171 (2004), 263-296.

[10] R. J. Briggs. Electron-stream interaction with plasmas. MIT Press, Cambridge, MA, 1964.

[11] V. Capasso, L. Maddalena. Convergence to equilibrium states for a reaction-diffusion system modelling the spatial spread of a class of bacterial and viral diseases. J. Math. Biology, 13 (1981), 173-184.

[12] X.-Y. Chen, J. K. Hale, B., Tan. Invariant foliations for $C^{1}$ semigroups in Banach spaces. J. Differential Equations, 139 (1997), 283-318.

[13] C. Chicone, Y. Latushkin. Evolution semigroups in dynamical systems and differential equations. Math. Surv. Monogr., 70, AMS, Providence, 1999.

[14] C. Chicone, Y. Latushkin. Center manifolds for infinite-dimensional nonautonomous differential equations. J. Differential Equations, 141 (1997), 356-399. 
[15] B. Deng. The existence of infinitely many traveling front and back waves in the FitzHugh-Nagumo equations. SIAM J. Math. Anal., 22 (1991), 653-679.

[16] K. Engel, R. Nagel. One-parameter semigroups for linear evolution equations. Springer, New York, 2000.

[17] J. W. Evans. Nerve axon equations. III. Stability of the nerve impulse. Indiana Univ. Math. J., 22 (1972), $577-593$.

[18] E. Feireisl. Bounded, locally compact global attractors for semilinear damped wave equations on $R^{N}$. Diff. Int. Eqns., 9 (1996), 1147-1156.

[19] A. Ghazaryan. Nonlinear stability of high Lewis number combustion fronts. Indiana Univ. Math. J., 58 (2009), 181-212.

[20] A. Ghazaryan, C. K. R. T. Jones. On the stability of high Lewis number combustion fronts. Discrete Contin. Dyn. Syst., 24 (2009), 809-826.

[21] A. Ghazaryan, Y. Latushkin, S. Schecter, A. J. de Souza. Stability of gasless combustion fronts in one-dimensional solids. Arch. Ration. Mech. Anal., 198 (2010), 981-1030.

[22] A. Ghazaryan, Y. Latushkin, S. Schecter. Stability of traveling waves for degenerate systems of reaction diffusion equations. Indiana Univ. Math. J., 60 (2011), 443-472.

[23] A. Ghazaryan, Y. Latushkin, S. Schecter. Stability of traveling waves for a class of reaction-diffusion systems that arise in chemical reaction models. SIAM J. Math. Anal., 42 (2010), 2434-2472.

[24] A. Ghazaryan, B. Sandstede. Nonlinear convective instability of Turing-unstable fronts near onset: a case study. SIAM J. Appl. Dyn. Syst., 6 (2007), 319-347.

[25] A. Ghazaryan, P. Simon, S. Schecter. Gasless combustion fronts with heat loss. To appear in SIAM J. Appl. Math.

[26] P. Gordon. Recent mathematical results on combustion in hydraulically resistant porous media. Math. Model. Nat. Phenom., 2 (2007), 56-76.

[27] K. P. Hadeler, M. A. Lewis. Spatial dynamics of the diffusive logistic equation with a sedentary compartment. Canadian Appl. Math. Quart, 10 (2002), 473-499.

[28] S. Heinze, B. Schweizer. Creeping fronts in degenerate reaction diffusion systems. Nonlinearity, 18 (2005), $2455-2476$.

[29] D. Henry. Geometric theory of semilinear parabolic equations. Lecture Notes in Mathematics vol. 840. Springer, New York, 1981.

[30] T. Kapitula, K. Promislow. An introduction to spectral and dynamical stability. Springer, New York, to appear.

[31] B. Kazmierczak, V. Volpert. Travelling waves in partially degenerate reaction-diffusion systems. Math. Model. Nat. Phenom., 2 (2007), 106-125.

[32] B. Kazmierczak, V. Volpert. Calcium waves in a system with immobible buffers as a limit of waves with a system with nonzero diffusion. Nonlinearity, 21 (2008), 71-96.

[33] B. Kazmierczak, V. Volpert. Mechano-chemical calcium waves in systems with immobile buffers. Arch. Mech., 60 (2008), 3-22 .

[34] B. Kazmierczak, V. Volpert. Travelling calcium waves in a system with non-diffusing buffers. Math. Methods and Models in Appl. Sci., 18 (2008), 883-912.

[35] G. Kreiss, H.-O. Kreiss, N. A. Petersson. On the convergence to steady state of solutions of nonlinear hyperbolicparabolic systems. SIAM J. Numer. Anal., 31 (1994), 157-1604.

[36] M. Kunze, G. Schneider. Exchange of stability and finite-dimensional dynamics in a bifurcation problem with marginally stable continuous spectrum. Z. Angew. Math. Phys., 55 (2004), 383-399.

[37] Y. Latushkin, B. Layton. The optimal gap condition for invariant manifolds. Discrete Contin. Dynam. Systems, 5 (1999), 233-268.

[38] Y. Latushkin, J. Prüss, R. Schnaubelt. Center manifolds and dynamics near equilibria of quasilinear parabolic systems with fully nonlinear boundary conditions. Discrete Contin. Dyn. Syst. Ser. B, 9 (2008), 595-633.

[39] Y. Li, Y. Wu. Stability of traveling front solutions with algebraic spatial decay for some autocatalytic chemical reaction systems. SIAM J. Math. Anal., 44 (2012), 1474-1521.

[40] S. Luckhaus, L. Triolo. The continuum reaction-diffusion limit of a stochastic cellular growth model. Rend. Mat. Acc. Lincei,, 15 (2004), 215-223.

[41] A. Lunardi. Analytic semigroups and optimal regularity in parabolic problems. Progr. Nonlin. Diff. Eqns. Appl. vol. 16, Birkhäuser, Basel, 1995.

[42] A. Mielke, G. Schneider. Attractors for modulation equations on unbounded domains-existence and comparison. Nonlinearity, 8 (1995), 743-768.

[43] J. D. Murray. Mathematical biology. I. An introduction and II. Spatial models and biomedical applications. Interdisciplinary Applied Mathematics vols. 17, 18. Springer, New York, 2002, 2003.

[44] S. Nii. Stability of travelling multiple-front (multiple-back) wave solutions of the FitzHugh-Nagumo equations. SIAM J. Math. Anal., 28 (1997), 1094-1112.

[45] R. L. Pego, M. I. Weinstein. Asymptotic stability of solitary waves. Comm. Math. Phys., 164 (1994), $305-349$.

[46] L. Roques. Study of the premixed flame model with heat losses. The existence of two solutions. European J. Appl. Math., 16 (2005), 741-765.

[47] J. Rottmann-Matthes. Computation and stability of patterns in hyperbolic-parabolic systems. Shaker Verlag, Aachen, 2010 .

[48] J. Rottman-Matthes. Linear stability of travelling waves in first-order hyperbolic PDEs. J. Dynam. Differential Equations, 23 (2011), 365-393.

[49] J. Rottmann-Matthes. Stability of parabolic-hyperbolic traveling waves. Dynamics of Part. Diff. Eqns., 9 (2012) $29-62$.

[50] J. Rottmann-Matthes. Stability and freezing of nonlinear waves in first order hyperbolic PDEs. J. Dynam. Differential Equations, 24 (2012), 341-367. 
[51] J. Rottmann-Matthes. Stability and freezing of waves in non-linear hyperbolic-parabolic systems. IMA J. Appl. Math., 77 (2012), 420-429.

[52] B. Sandstede. Stability of $N$-fronts bifurcating from a twisted heteroclinic loop and an application to the FitzHughNagumo equation. SIAM J. Math. Anal., 29 (1998), 183-207.

[53] B. Sandstede. Stability of traveling waves. In Handbook of dynamical systems, vol. 2, 983-1055. North-Holland, Amsterdam, 2002.

[54] B. Sandstede, A. Scheel. Absolute and convective instabilities of waves on unbounded and large bounded domains. Phys. D, 145 (2000), 233-277.

[55] D. H. Sattinger. On the stability of waves of nonlinear parabolic systems. Adv. Math., 22 (1976), 312-355.

[56] G. Sell, Y. You. Dynamics of evolutionary equations. Applied Mathematical Sciences vol. 143, Springer, 2002.

[57] P. Simon, J. Merkin, S. Scott. Bifurcations in non-adiabatic flame propagation models. Focus on Combustion Research (2006), 315-357.

[58] P. Simon, S. Kalliadasis, J.H. Merkin, S.K. Scott. Inhibition of flame propagation by an endothermic reaction. IMA J. Appl. Math., 68 (2003), 537-562.

[59] P. Simon, S. Kalliadasis, J.H. Merkin, S.K. Scott. Stability of flames in an exothermic-endothermic system. IMA J. Appl. Math., 69 (2004), 175-203.

[60] P. Simon, S. Kalliadasis, J.H. Merkin, S.K. Scott. On the structure of the spectra for a class of combustion waves. J. Math. Chem., 35 (2004), 309-328.

[61] P. Simon, J. Merkin, S. Scott. Bifurcations in non-adiabatic flame propagation models. Focus on Combustion Research (2006), 315-357.

[62] J.-C. Tsai. Global exponential stability of traveling waves in monotone bistable systems. Discrete Contin. Dyn. Syst., 21 (2008), 601-623.

[63] J.-C. Tsai, J. Sneyd. Existence and stability of traveling waves in buffered systems. SIAM J. Appl. Math., 66 (2005), $237-265$.

[64] J.-C. Tsai, W. Zhang, V. Kirk, J. Sneyd. Traveling waves in a simplified model of calcium dynamics. SIAM J. Appl. Dyn. Systems, 11 (2012), 1149-1199.

[65] J. M. A. M. van Neerven. The asymptotic behavior of semigroups of linear operators. Operator Theory: Advances and Applications vol. 88. Birkhäuser, Basel, 1996.

[66] W. van Saarloos. Front propagation into unstable states. Phys. Rep., 386 (2003), 29-222.

[67] F. Varas, J. Vega. Linear stability of a plane front in solid combustion at large heat of reaction. SIAM J. Appl. Math., 62 (2002), 1810-1822.

[68] V. Volpert, V. Vougalter. Emergence and propagation of patterns in nonlocal reaction-diffusion equations arising in the theory of speciation. In Dispersal, individual movement and spatial ecology: a mathematical perspective. Lecture Notes in Mathematics vol. 2071, 331-354. Springer, New York, 2013.

[69] E. Yanagida. Stability of travelling front solutions of the FitzHugh-Nagumo equations. Math. Comput. Modelling, 12 (1989), 289-301.

[70] K. Zumbrun, P. Howard. Pointwise semigroup methods and stability of viscous shock waves. Indiana Univ. Math. J., 47 (1998), 741-871. 